\title{
Concomitant stenting of the patent ductus arteriosus and radiofrequency valvotomy in pulmonary atresia with intact ventricular septum and intermediate right ventricle: Early in-hospital and medium-term outcomes
}

\author{
Mazeni Alwi, MRCP, ${ }^{\mathrm{a}}$ Kok-Kuan Choo, MRCP, ${ }^{\mathrm{a}}$ Nomee A. M. Radzi, MRCPCH, ${ }^{\mathrm{a}}$ Hasri Samion, MD, ${ }^{\mathrm{a}}$ \\ Kiew-Kong Pau, FRCS, ${ }^{\text {b }}$ and Chee-Chin Hew, FRCS ${ }^{\mathrm{b}}$
}

\begin{abstract}
Objectives: Our objective was to determine the feasibility and early to medium-term outcome of stenting the patent ductus arteriosus at the time of radiofrequency valvotomy in the subgroup of patients with pulmonary atresia with intact ventricular septum and intermediate right ventricle.
\end{abstract}

\begin{abstract}
Background: Stenting of the patent ductus arteriosus and radiofrequency valvotomy have been proposed as the initial intervention for patients with intermediate right ventricle inasmuch as the sustainability for biventricular circulation or $1 \frac{1}{2}$-ventricle repair is unclear in the early period.
\end{abstract}

\begin{abstract}
Methods: Between January 2001 and April 2009, of 143 patients with pulmonary atresia and intact ventricular septum, 37 who had bipartite right ventricle underwent radiofrequency valvotomy and stenting of the patent ductus arteriosus as the initial procedure. The mean tricuspid valve $\mathrm{z}$-score was $-3.8 \pm 2.2$ and the mean tricuspid valve/mitral valve ratio was $0.62 \pm 0.16$.
\end{abstract}

\begin{abstract}
Results: Median age was 10 days (3-65 days) and median weight $3.1 \mathrm{~kg}(2.4-4.9 \mathrm{~kg})$. There was no procedural mortality. Acute stent thrombosis developed in 1 patient and necessitated emergency systemic-pulmonary shunt. There were 2 early in-hospital deaths owing to low cardiac output syndrome. One late death occurred owing to right ventricular failure after the operation. Survival after the initial procedure was $94 \%$ at 6 months and $91 \%$ at 5 years. At a median follow-up of 4 years (6 months to 8 years), 17 (48\%) attained biventricular circulation with or without other interventions and $9(26 \%)$ achieved 1 1/2-ventricle repair. The freedom from reintervention was $80 \%, 68 \%, 58 \%$, and $40 \%$ at $1,2,3$, and 4 years, respectively.
\end{abstract}

Conclusions: Concomitant stenting of the patent ductus arteriosus at the time of radiofrequency valvotomy in patients with pulmonary atresia with intact ventricular septum and intermediate right ventricle is feasible and safe with encouraging medium-term outcome. (J Thorac Cardiovasc Surg 2011;141:1355-61)

The wide anatomic spectrum of pulmonary atresia with intact ventricular septum (PAIVS) is widely recognized and thus management strategy needs to be tailored. ${ }^{1-3}$ Patients with membranous atresia and well-developed, "tripartite" right ventricle (RV) generally respond well with radiofrequency-assisted valvotomy and balloon dilatation (RFV) or alternatively surgical valvotomy in the neonatal period. This may be the only procedure required (2-ventricle circulation). ${ }^{4}$ At the other end of the anatomic spectrum are patients with severe RV hypoplasia, absent infundibulum (muscular atresia), often associated with major RVcoronary communications (sinusoids). This is essentially

\footnotetext{
From the Departments of Paediatric Cardiology ${ }^{\mathrm{a}}$ and Cardiothoracic Surgery, ${ }^{\mathrm{b}}$ Institut Jantung Negara (National Heart Institute), Kuala Lumpur, Malaysia.

Disclosures: Authors have nothing to disclose with regard to commercial support.

Received for publication May 14, 2010; revisions received Aug 3, 2010; accepted for publication Aug 30, 2010; available ahead of print Jan 12, 2011.

Address for reprints: Mazeni Alwi, MRCP, Department of Paediatric Cardiology, Institut Jantung Negara (National Heart Institute), 145, Jalan Tun Razak, 50400

Kuala Lumpur, Malaysia (E-mail: mazeni@ijn.com.my).

0022-5223/\$36.00

Copyright (c) 2011 by The American Association for Thoracic Surgery

doi:10.1016/j.jtcvs.2010.08.085
}

an anomaly for single-ventricle repair with systemicpulmonary shunt as the initial palliation. ${ }^{5}$ Of interest are patients with intermediate anatomy in whom the atresia is membranous and the infundibulum is fairly well developed but the RV is moderately hypoplastic; often the RV is described as "bipartite." At presentation it is unclear whether the RV would ultimately be able to independently support the pulmonary circulation. ${ }^{6}$

Although RFV is effective in opening up the pulmonary valve and decompressing the $\mathrm{RV}$, a significant proportion of patients, especially those with moderate RV hypoplasia, require a systemic-pulmonary shunt within days of the procedure. ${ }^{7,8}$ The objectives of this study were to determine the feasibility and safety of concomitant stenting of the patent ductus arteriosus (PDA) at the time of RFV in the subgroup of patients with intermediate RV and, second, to review the medium-term outcome of this subgroup.

\section{METHODS}

\section{Definition and Patient Selection}

All patients with PAIVS underwent detailed echocardiography and this served as the basis for the initial strategy, which consist mainly of 


$$
\begin{aligned}
\text { Abbreviations and Acronyms } \\
\text { ASD }=\text { atrial septal defect } \\
\text { MV }=\text { mitral valve } \\
\text { PAIVS = pulmonary atresia with intact } \\
\text { ventricular septum } \\
\text { PDA }=\text { patent ductus arteriosus } \\
\text { RV }=\text { right ventricle } \\
\text { RVF }=\text { radiofrequency-assisted valvotomy and } \\
\text { RVOT }=\text { balloon dilatation } \\
\text { TV }=\text { tricuspid valve }
\end{aligned}
$$

catheter-based therapies. The tricuspid valve (TV) and mitral valve (MV) diameters were measured in diastole. The TV z-score was derived from the normogram by Rowlatt, Rimoldi, and Lev, ${ }^{9}$ and TV z-score was calculated. ${ }^{10}$ The RV cavity was also described as tripartite, bipartite, or unipartite, depending on the number of parts of the RV (inlet, apical trabecular, and outlet) not obliterated by intracavity muscular overgrowth. Particular attention was paid to whether there was a well-formed infundibulum that ended in a membranous atresia or was obliterated by muscles (muscular atresia). TV regurgitation and its severity, if present, were noted as well as RV-coronary communications. Patients with "good" RV are those with a TV z-score of more than -2.5 , a TV/MV ratio of 0.75 or more, and membranous atresia with a well-formed infundibulum in which the $\mathrm{RV}$ cavity was described as tripartite. The RV volume was not measured quantitatively. ${ }^{10}$ Patients with a "severely diminutive" RV were defined as those having a TV z-score of less than -5.0 , a TV/MV ratio of less than 0.5 , and an RV cavity described as unipartite, in which only the inlet component was present and the infundibulum was absent or slitlike. These 2 subgroups were excluded from the study. Also excluded were patients with branch pulmonary artery stenosis, usually the left at the site of ductal insertion. The subjects of this study, termed as having "intermediate" RV morphology, were those with a TV z-score of -2.5 to -5.0 and/or a TV/MV ratio of 0.50 to 0.75 , with the RV described as "bipartite," where apart from the inlet component, a well-formed infundibulum ends in a membranous atresia but the apical trabecular component is absent or attenuated. The decision for recruitment into the study was made by a consensus of 2 of the authors (M.A. and H.S.). Ethics committee approval was obtained.

\section{RFV and PDA Stenting, Postprocedure Care}

All patients were subjected to cardiac catheterization under general anesthesia with a view toward catheter-based intervention. Patients with a good RV underwent RFV only. Patients with a severely diminutive (unipartite) RV received PDA stenting only if the PDA was suitable; otherwise, they were referred for systemic-pulmonary shunt. The study patients, those with an intermediate RV, received RFV and concomitant PDA stenting. Prostaglandin $\mathrm{E}_{1}$ was discontinued the evening before the procedure to ensure the PDA was restrictive. Informed consent was obtained. Baseline hemodynamic data and oxygen saturation were obtained. RV angiogram was performed to delineate the RV cavity, particularly the infundibulum, the valve, and subvalvular area, and to document RV-coronary communications and the possibility of RV-dependent coronary circulation. RV decompression with RFV was not performed in the presence of RVdependent coronary circulation. Aortic arch angiography was performed to delineate the PDA and the pulmonary artery anatomy for the purpose of PDA stenting. RFV and PDA stenting were performed using techniques and equipment as $\mathrm{we}^{4,11}$ previously described. Coronary stents were used and the sizes chosen were $3.5 \mathrm{~mm}$ for patients weighing 2.5 to $2.9 \mathrm{~kg}$, $4.0 \mathrm{~mm}$ for those weighing 3.0 to $3.5 \mathrm{~kg}$, and $4.5 \mathrm{~mm}$ for those weighing more than $3.5 \mathrm{~kg}$. The stent length was according to that of the PDA. All procedures were done by the transfemoral route. PDA stenting was not performed if branch pulmonary artery stenosis at the site of ductal insertion was shown by angiography. Early in the series, balloon atrial septostomy was also performed if the patent foramen ovale was restrictive, but this was subsequently omitted because we believed that a large interatrial communication would reduce flow to the TV and impair RV growth. After the procedure, the patients were returned to the intensive care unit for continued ventilation and inotropic support as necessary. Heparin infusion was given for 2 days and oral aspirin started the following day and continued until the end point was reached.

\section{Immediate Early Outcome, Follow-up, and Subsequent Procedures}

Patients were discharged from the intensive care unit if they could be extubated, could maintain an oxygen saturation greater than $75 \%$ in air, and could maintain stable hemodynamics without intropic support. Early reintervention is defined as the patient requiring a systemic-pulmonary shunt within 30 days of the procedure owing to oxygen saturation less than $70 \%$ or requiring other surgical/catheter procedures.

After discharge, the patients were reviewed at 1 month, 3 months, and then every 6 months. The degree of cyanosis was assessed clinically. The major tool for evaluation was echocardiography, focusing on the growth of the RV by measuring the TV and MV, and a general assessment of RV cavity, in particular the apical trabecular component. Tricuspid regurgitation, if present, was monitored as well as any residual or recurrence of valvular or subvalvular stenosis. The flow through the ductal stent was noted.

The end points were death before subsequent procedures and, for the surviving patients, attainment of either 2-ventricle circulation or $1 \frac{1}{2}$-ventricle circulation. The patients were described as having 2 -ventricle circulation and no further treatment was deemed necessary if there was no obvious clinical cyanosis, the echocardiographic evaluation fit the description of tripartite RV with a gradient of $40 \mathrm{~mm} \mathrm{Hg}$ or less across the RV outflow tract (RVOT), absent or minimal flow through the PDA, and minimal shunt through the patent foramen ovale. Significant additional problems, if present, such as RVOT obstruction owing to subvalvular muscular overgrowth or hypoplastic pulmonary annulus, shunts through the ASD or PDA, and tricuspid regurgitation were addressed before being categorized as having attained the 2-ventricle circulation.

Patients who remained cyanotic despite good antegrade flow and whose $\mathrm{RV}$ growth remained inadequate after 36 to 60 months were subjected to $1 \frac{1}{2}$-ventricle circulation by having bidirectional cavopulmonary shunt. Prior cardiac catheterization was performed partly to specifically look for branch left pulmonary artery stenosis as a possible late complication of PDA stenting. At the time of the bidirectional cavopulmonary procedure, the ASD was closed, the PDA stent divided, and additional problems, if present, such as TV regurgitation, RVOT obstruction, pulmonary annular hypoplasia, and branch left pulmonary artery stenosis were addressed accordingly. Patients who had not reached either end point were considered as "awaiting."

\section{Analysis of Data}

The SPSS statistical program for Windows, version 17.0 (SPSS, Inc, Chicago, Ill), was used for data analysis. Data was expressed as mean \pm $\mathrm{SD}$, median (range), and frequency (percentage). Survival and freedom from reinterventions were determined by the Kaplan-Meier method. Reintervention was defined as either catheter-based intervention or surgery.

\section{RESULTS}

Between January 2001 and April 2009, 143 patients with PAIVS were referred to our institution. Forty-five patients had good RV (tripartite) and underwent RFV only. 
TABLE 1. Demographic, echocardiographic, periprocedural, and follow-up data

\begin{tabular}{lc}
\hline Median age (d) & $10(3-65)$ \\
Median weight $(\mathrm{kg})$ & $3.1(2.6-4.9)$ \\
Mean TV/MV ratio & $0.62 \pm 0.16$ \\
Mean z-score of TV annulus & $-3.8 \pm 2.2$ \\
Mean procedure time (min) & $111 \pm 39$ \\
Median ventilation days (d) & $1(1-15)$ \\
Median length of hospital stay (d) & $8(4-23)$ \\
Median follow up (y) & $4(1 / 2-8.2)$ \\
\hline
\end{tabular}

Sixty-one patients had severely diminutive RV (unipartite) and received PDA stenting only. Thirty-seven patients, who formed the subjects of this study (ie, intermediate RV size [bipartite]), received RFV and elective PDA stenting as the initial procedure. Early in the series, $8(22 \%)$ patients received additional balloon atrial septostomy.

The median age at procedure was 10 days (3-65 days) and the mean weight was $3.1 \mathrm{~kg}(2.6-4.9 \mathrm{~kg})$. The TV z-score was $-3.8 \pm 2.2$. The TV/MV ratio was $0.62 \pm$ 0.16 (Table 1).

In all patients the RV cavity was described as bipartite, having definite inlet and infundibular components with membranous atresia, whereas the apical trabecular component was severely attenuated. Moderate-to-severe tricuspid regurgitation was present in $12(32 \%)$ patients. Major sinusoids were present in $3(8.1 \%)$, but none had RV-dependent coronary circulation.

The mean procedural time was $111 \pm 39$ minutes. The median duration of ventilatory support was 1 day (1-15 days). The median length of hospital stay was 8 days (4-23 days) (Table 1).

\section{Mortality and Complications}

There was no procedural mortality. Two $(5 \%)$ patients had transient supraventricular tachycardia. One patient had acute stent thrombosis within 24 hours, necessitating systemic-pulmonary shunt. Loss of femoral arterial pulse occurred in $5(14 \%)$ and was successfully treated with heparin and streptokinase.

There were 2 early in-hospital deaths owing to low cardiac output at 10 and 12 days after the procedure. One of these patients had major sinusoids and the other had pulmonary overcirculation. One late death was due to RV failure after RVOT reconstruction surgery. Survival after the initial procedure was $94 \%$ at 6 months and remained at $91 \%$ from 1 to 4 years (Figure 1).

\section{Follow-up}

At a median follow-up of 4 years (6 months to 8.2 years), $8(23 \%)$ patients had not reached the study end point (follow-up <24 months; ie, "awaiting"). Seventeen $(48 \%)$ attained biventricular circulation, 8 of whom required reinterventions: transcatheter closure of ASD $(\mathrm{n}=2)$, repeat balloon pulmonary valvuloplasty $(\mathrm{n}=1)$, surgical RVOT reconstruction $(\mathrm{n}=4)$, and PDA restenting and balloon pulmonary valvuloplasty at 3 months, followed by RVOT reconstruction at 14 months $(n=1)$. Nine $(26 \%)$ patients did not achieve sufficient RV growth and received bidirectional cavopulmonary shunt, ASD closure, and PDA stent division (1 1/2-ventricle repair), of whom $3(33 \%)$ also received RVOT reconstruction (Figure 1). No patient had left pulmonary artery stenosis.

The freedom from reintervention was $80 \%, 68 \%, 58 \%$, and $40 \%$ at 1, 2, 3, and 4 years, respectively (Figure 2).

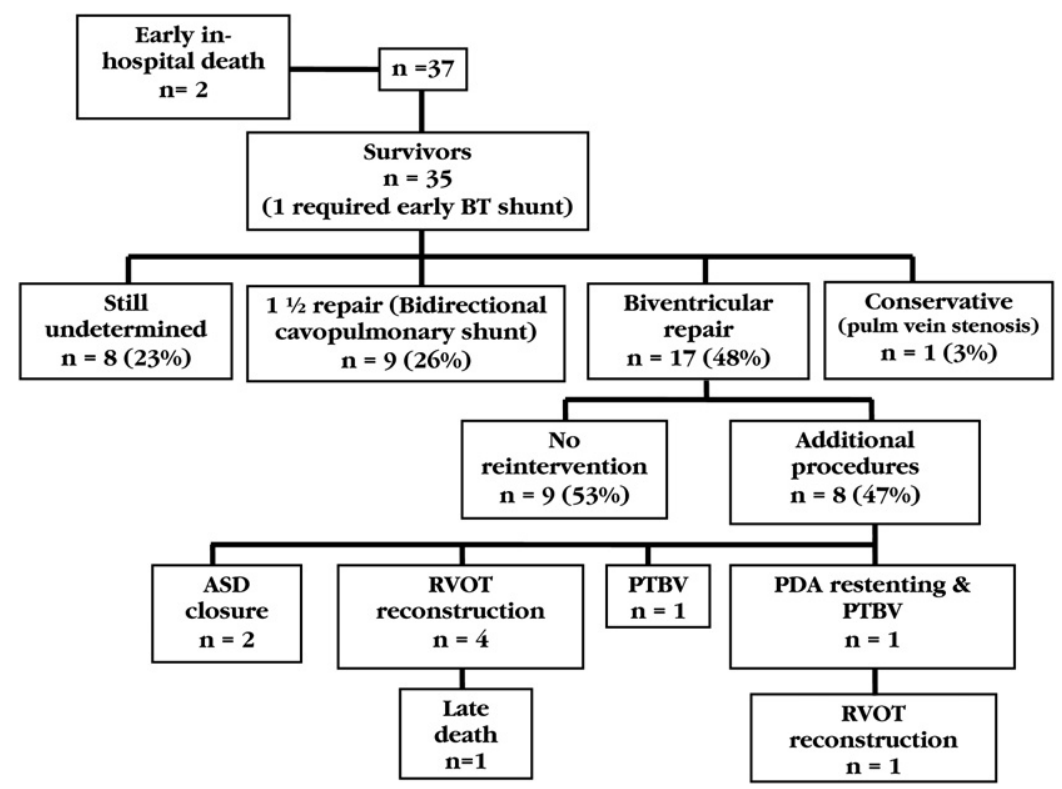

FIGURE 1. Outcome of patients with intermediate right ventricle $(R V) . B T$, Blalock-Taussig shunt; $A S D$, atrial septal defect; $R V O T$, right ventricular outflow tract; $P T B V$, percutaneous transcatheter balloon valvuloplasty; $P D A$, patent ductus arteriosus. 


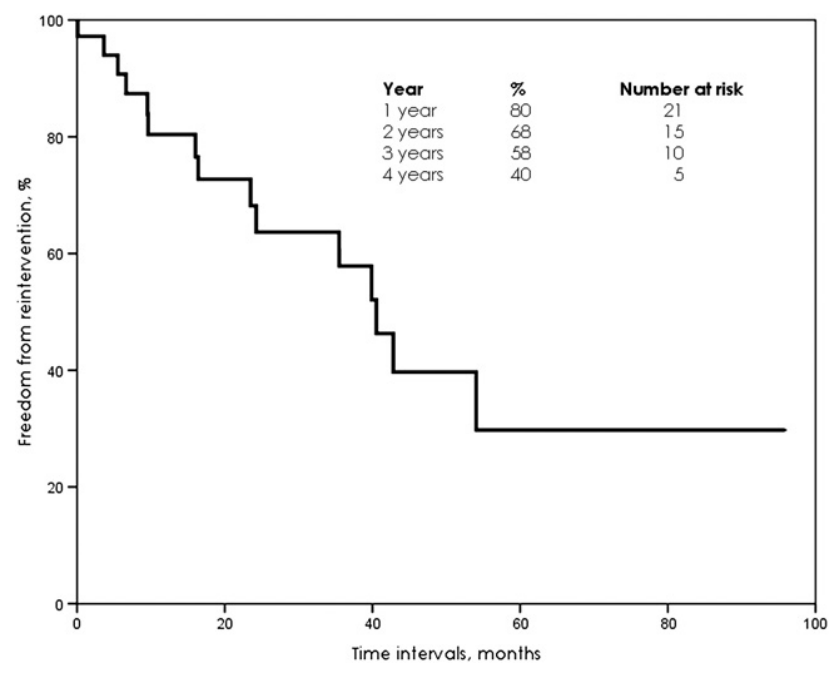

FIGURE 2. Freedom from reintervention (overall).

However, for those who attained biventricular circulation, the freedom from reintervention was $77 \%, 70 \%, 70 \%$, and $61 \%$ at $1,2,3$, and 4 years, respectively (Figure 3 ).

The mean TV score at presentation for those who attained biventricular circulation and 1/1/2-ventricle repair was $-2.8 \pm$ 1.8 and $-4.1 \pm 2.3$, respectively.

\section{DISCUSSION}

The wide variation of RV morphology in PAIVS has long been recognized and management algorithms have largely been based on this. ${ }^{1-3}$ At one end of the morphologic spectrum are those with a good RV in whom the 3 components of the RV cavity are reasonably well developed - the inlet, apical trabecular, and outlet or infundibulum-even if the RV in general is smaller than that of the normal population. The atresia in this group of patients is membranous in nature. This is the group with the most favorable outcome, comprising $50 \%$ to $60 \%$ of patients. RV decompression and establishing unobstructed antegrade flow into the pulmonary circulation by surgical valvotomy or RFV in the neonatal period is often the only procedure required, the latter being the favored method of treatment today. ${ }^{4}$

At the other end are those with severely diminutive RV with obliteration of the apical trabecular and infundibular cavity. These patients are inevitably destined for the Fontan track. $^{3,5,12}$ Inasmuch as they are ductus dependent, an initial palliation with systemic-pulmonary shunt is required. PDA stenting, being less invasive, is an attractive alternative to surgical shunt. This is particularly suited to PAIVS, in which the ductus tends to be more amenable to stenting compared with other forms of cyanotic heart disease. ${ }^{11,13,14}$ This is our preferred mode of initial management.

However, a significant proportion of patients do not exhibit RV features that clearly suggest either the favorable

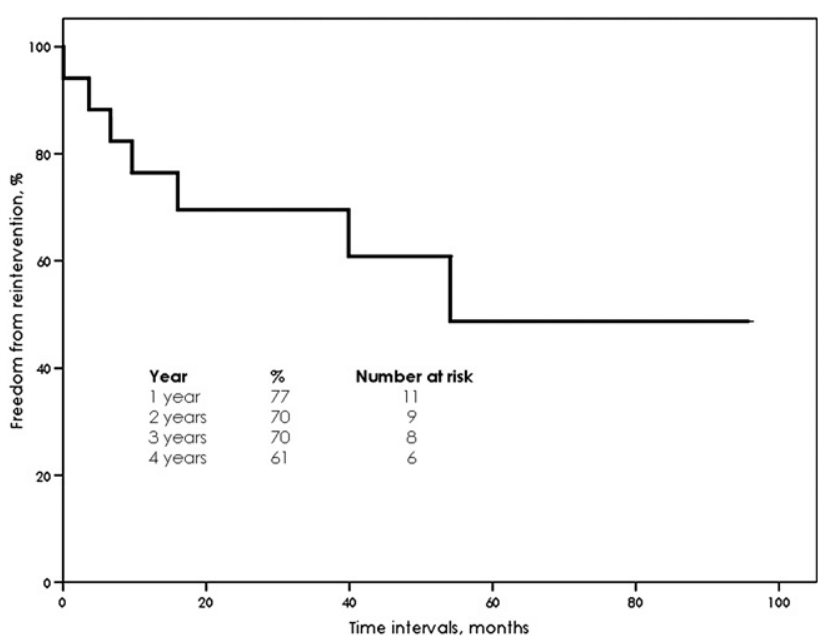

FIGURE 3. Freedom from reintervention (biventricular circulation).

2-ventricle eventual outcome or the less favorable singleventricle pathway. In our algorithm, this subgroup of patients was designated "intermediate." ${ }^{6}$ The characteristic features on echocardiography that define this subgroup are $\mathrm{TV} \mathrm{z}$-score -2.5 to $-5.0, \mathrm{MV} / \mathrm{TV}$ ratio 0.5 to 0.75 , and an RV cavity that has a severely attenuated apical trabecular component but with a reasonably well-developed infundibulum that ends with a membranous valvular atresia, "bipartite RV."

Somewhat out of this continuum of RV morphology are the rare patients with Ebstein anomaly, who have a markedly dilated, thin-walled, poorly functioning RV with severe tricuspid regurgitation. ${ }^{2}$ Even today the outlook is very poor, with or without intervention.

Catheter-based therapies have markedly altered the management of PAIVS. RFV is currently the preferred method of initial management, achieving results comparable with if not superior to conventional surgery. ${ }^{4,7,8}$ The PDA in this condition tends to arise obtusely from the descending aorta to insert onto the dome of the main pulmonary artery rather than onto the proximal left pulmonary artery, making PDA stenting an attractive alternative to systemic-pulmonary shunt. Transcatheter device closure of shunts at ASD or PDA levels can be done as the final procedure. Radical surgery, such as RVOT reconstruction in the neonatal period, is associated with high mortality and morbidity. ${ }^{12}$ However, surgery remains an important part of management of PAIVS, especially for those with severely diminutive RV who require the Fontan operation. ${ }^{5,12,15,16}$ In patients with intermediate RV that remains incapable of independently supporting the pulmonary circulation, the bidirectional cavopulmonary

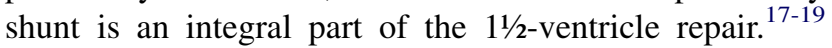
Although data are currently lacking, we are of the opinion

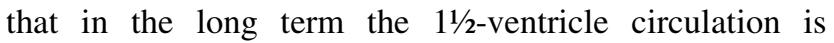


preferable to the Fontan circulation inasmuch as some of the major late complications of the Fontan operation are attributed to the absence of pulsatile flow. This is our basis for this management strategy for those with borderline, "intermediate" RV. Surgery may also be additionally required in patients with fixed RVOT obstruction and severe tricuspid regurgitation in both the 2-ventricle and 1/2-ventricle pathways.

\section{Less Ideal RV Size and the Basis for "Prophylactic" PDA Stenting}

The focus of our interest is on the subgroup of patients with borderline or "intermediate" RV, one that is difficult to determine at the time of presentation whether it would eventually be capable of sustaining the pulmonary circulation (2-ventricle) or have to be supported by a bidirectional cavopulmonary shunt (11/2-ventricle). The initial management of patients with intermediate $\mathrm{RV}$ as defined herein is somewhat less clear-cut than those with good tripartite $\mathrm{RV}$ or those with severely diminutive RV.

In the surgical era, closed valvotomy or transannular patch with concomitant systemic-pulmonary shunt is a popular management strategy for all patients with patent infundibulum. ${ }^{20,21}$ It was not uncommon for patients to require such a shunt within days of surgical valvotomy alone owing to unacceptably low arterial oxygen tension, hence the concept of "prophylactic" systemic-pulmonary shunt at the time of valvotomy. A parallel situation was encountered in the era of transcatheter management with RFV. In a relatively large series of PAIVS patients with seemingly good RV (mean TV z-score -1.33 ), 14 of 27 patients who successfully underwent RFV required the construction of a systemic-pulmonary shunt 2 to 24 days after the initial procedure. ${ }^{8}$ At about the same time, Agnoletti and associates ${ }^{7}$ reported that after successful perforation in 33 patients (medium TV z-score -1.2), 12 required a Blalock-Taussig shunt and 8 required a Blalock-Taussig shunt and RVOT reconstruction 1 to 30 days after the initial procedure, with a bias toward those with more negative TV z-score requiring early surgical reintervention. This was also reflected in our own experience, although the proportion of patients who required systemic-pulmonary shunt or PDA stenting within 14 days of RFV was lower. ${ }^{22}$ On the basis of these findings, in patients who are identified as having less ideal RV size, that is, the intermediate RV group, there is merit in incorporating PDA stenting into RFV as initial management. The rationale is to avoid reintervention, either systemic-pulmonary shunt or PDA stenting, within days of RFV by maintaining the PDA as an additional source of pulmonary flow until there is sufficient RV "growth" (2-ventricle) or otherwise where there is a need then for bidirectional cavopulmonary shunt $\left(1 \frac{1}{2}\right.$-ventricle). That PDA stenting in the current era has shown results that are comparable with those of Blalock-
Taussig shunt in terms of safety and efficacy provides further rationale for this strategy. ${ }^{11,13,14}$ On the basis of data from these 3 relatively large series and established surgical practice, the argument for concomitant PDA stenting in all patients with PAIVS undergoing RFV does seem persuasive. However, PDA stenting as an alternative to systemic-pulmonary shunt is a newly introduced procedure, it is technically demanding, experience is limited, and the full extent of its late complications is yet to be known. Hence, this treatment strategy cannot be universally recommended for all PAIVS patients, but applying it for a selected group is justifiable.

Our results for this selected group of PAIVS patients showed that this combined procedure was well tolerated and the immediate outcome encouraging as reflected in the intensive care unit stay. There were no deaths or major acute complications owing to the PDA stenting itself, except that in 1 patient acute stent thrombosis developed within 24 hours, requiring emergency systemic-pulmonary shunt.

From our experience, PDA stenting is a reasonable alternative to the Blalock-Taussig shunt in a selected group of patients. However, one needs to be mindful of the possible complications, such as acute stent thrombosis, aggressive neointimal proliferation causing early stent flow restriction, and late branch pulmonary artery stenosis in those in whom the ductus is inserted at the left pulmonary artery. Hence, this has to be excluded at the initial evaluation. ${ }^{11}$ In PAIVS, RFV and PDA stenting may cause overcirculation and myocardial ischemia, in particular in those with coronary sinusoids.

In this series, major late complications of PDA stenting were not encountered and a stented PDA did not pose a major surgical challenge at the time of bidirectional cavopulmonary shunt, where the stent was crushed, sutured with 5-0 Prolene polypropylene (Ethicon, Inc, Somerville, NJ), and divided.

As anticipated, not all patients attained 2-ventricle circulation, where normal oxygen saturation is achieved with the RV supporting the entire pulmonary circulation and all shunts abolished. It was also not surprising that $50 \%$ of patients who did attain the 2-ventricle end point required reinterventions in the form of RVOT reconstruction, ASD closure, and TV repair given that they started off with less ideal morphology. Twenty-six percent of patients did not achieve sufficient RV "growth" after 3.7 years \pm 2.9 years and received bidirectional cavopulmonary shunt, ASD closure, and PDA stent division (11/2-ventricle repair).

\section{RV "Growth"}

The potential for growth of the RV after decompression and establishment of forward flow is difficult to predict. There have been many published series documenting anatomic growth of the RV, in part contributed by pulmonary 

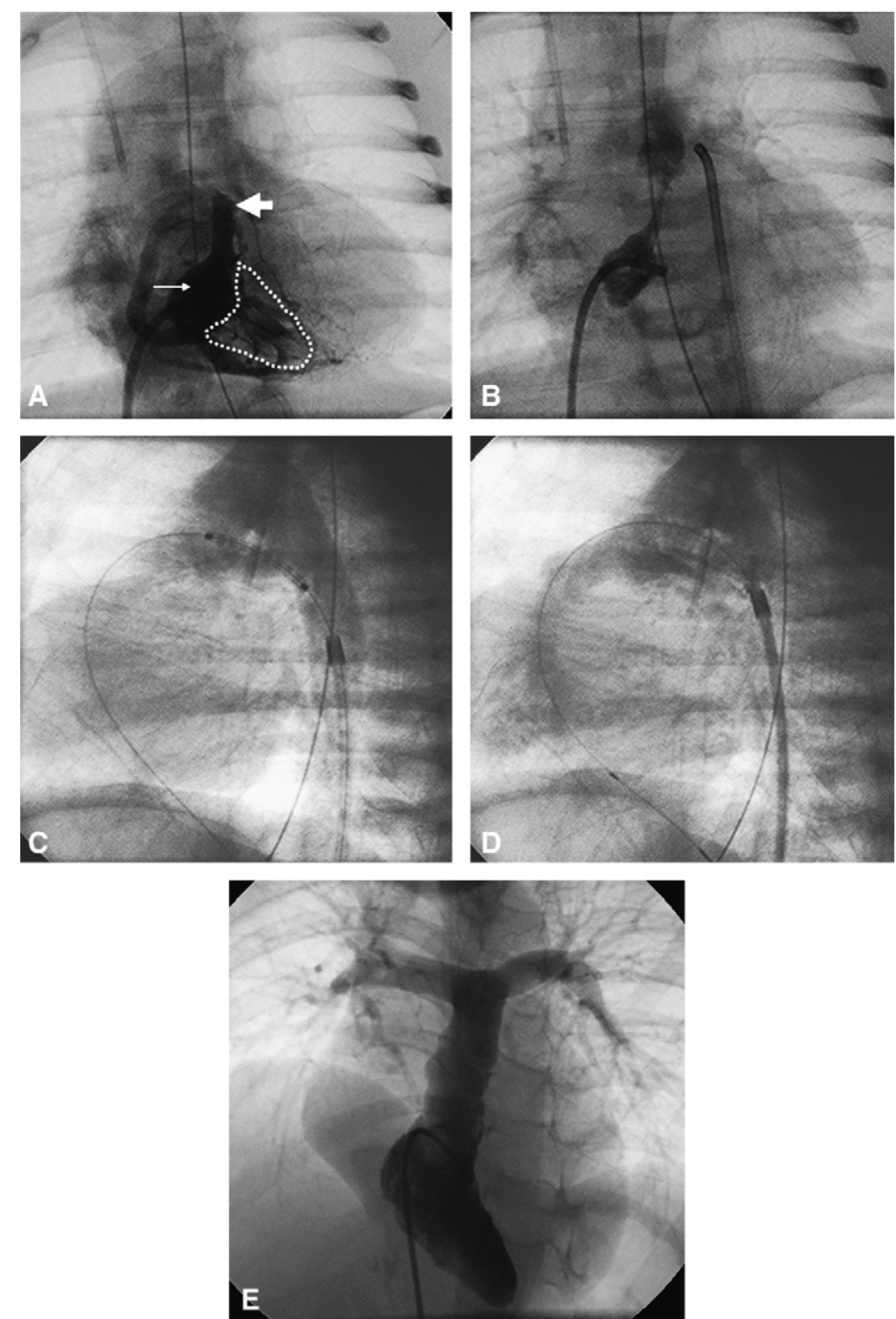

FIGURE 4. A, Right ventricular (RV) handshot showing bipartite RV with fairly well-developed infundibulum with membranous atresia (thick arrow) and inlet component (thin arrow). Muscle-bound apical trabecular component of RV is the area within dotted lines. Except for the intertrabecular recesses, the cavity is virtually obliterated. Membranous atresia seen. RV/aortic pressures $=153: 73 \mathrm{~mm} \mathrm{Hg}$. B, RV angiogram after radiofrequency valvotomy and balloon dilatation. Transient reactive spasm reduces RV outflow tract cavity. C, Patent ductus arteriosus crossed with a balloon-mounted coronary stent retrogradely. D, Stent expanded and covering the full length of the patent ductus arteriosus. E, RV angiogram 4 years after radiofrequency-assisted valvotomy and PDA stenting showing a well developed RV, unobstructed pulmonary blood flow, no branch pulmonary artery stenosis, and regression of muscular overgrowth resulting in a well-formed cavity of apical component of RV. Excellent overall growth of RV; "tripartite" end state. The patent ductus arteriosus stent is hardly visible.

regurgitation after valvotomy and regression of muscular hypertrophy after RV decompression. ${ }^{20,23,24}$ This is also illustrated by 1 of our patients (Figure $4, A-E$ ). On the other hand, there seem at times, overly optimistic expectations on the capacity for the RV to grow inasmuch as there are also data that showed no significant catch-up anatomic growth of the TV. However, even if there is no catch-up growth, some patients were still able to attain 2-ventricle circulation despite the subnormal RV size. ${ }^{8,25}$ It is thus difficult to determine the size of the RV that will contribute to a successful 2-ventricle circulation. It appears that normal $\mathrm{TV}$ growth is not mandatory to have an RV capable of sustaining the pulmonary circulation. "Physiologic" growth, that is, improved compliance, is of greater import 
than anatomic growth. In the face of these uncertainties, RV decompression provides the opportunity for the RV to attain its maximal potential for "growth," both anatomic and physiologic. This can be done less invasively by RFV, and PDA stenting helps support the pulmonary circulation in the interim until either of the end points is reached.

\section{CONCLUSIONS}

Concomitant PDA stenting at the time of RFV in PAIVS patients with intermediate RV size is feasible and safe. This treatment strategy largely obviated the need for emergency procedures to augment pulmonary blood flow although acute stent thrombosis may occur in a small percentage.

A quarter of patients with intermediate RV eventually required bidirectional cavopulmonary shunt (11/2-ventricle), whereas among those who attained biventricular circulation, a significant proportion required additional procedures. Concomitant with RFV, PDA stenting should be considered an integral part of the initial management in PAIVS with intermediate RV. However, inasmuch as PDA stenting is a relatively new procedure and PAIVS is an uncommon disease, this management strategy warrants wider, multi-institutional study.

We greatly appreciate the help of Muhaneeza Hanafiah in the preparation of the manuscript and Mohd Faizal Ramli for his assistance in the statistical analysis.

\section{References}

1. Daubeney PEF, Delany DJ, Anderson RH, Sandor GG, Slavik Z, Keeton BR, et al. Pulmonary atresia with intact ventricular septum: range of morphology in a population-based study. J Am Coll Cardiol. 2002;39:1670-9.

2. Dyamenahalli U, McCrindle BW, McDonald C, Trivedi KR, Smallhorn JF, Benson LN, et al. Pulmonary atresia with intact ventricular septum: management of, and outcomes for, a cohort of 210 consecutive patients. Cardiol Young. 2004; 14:299-308.

3. Ashburn MD, Blackstone EH, Wells WJ, Jonas RA, Pigula FA, Manning PB, et al. Determinants of mortality and type of repair in neonates with pulmonary atresia and intact ventricular septum. J Thorac Cardiovasc Surg. 2004;127:1000-8.

4. Alwi M, Geetha K, Bilkis AA, Lim MK, Hasri S, Haifa AL, et al. Pulmonary atresia with intact ventricular septum percutaneous radiofrequency-assisted valvotomy and Blalock Taussig shunt. J Am Coll Cardiol. 2000;35:468-76.

5. Jahangiri M, Zurakowski D, Bichell D, Mayer JE, del Nido PJ, Jonas RA. Improved results with selective management in pulmonary atresia with intact ventricular septum. J Thorac Cardiovasc Surg. 1999;118:1046-55.

6. Alwi M. Management algorithm in pulmonary atresia with intact ventricular septum. Catheter Cardiovasc Interv. 2006;67:679-86.

7. Agnoletti G, Piechaud JF, Bonhoeffer P, Aggoun Y, Massih TA, Boudjemline Y, et al. Perforation of the atretric pulmonary valve. J Am Coll Cardiol. 2003;41: 1399-403.
8. Humpl T, Söderberg B, McCrindle BW, Nykanen DG, Freedom RM, Williams WG, et al. Percutaneous balloon valvotomy in pulmonary atresia with intact ventricular septum-impact on patient care. Circulation. 2003;108: 826-32.

9. Rowlatt JF, Rimoldi M, Lev M. The quantitative anatomy of the normal child's heart. Pediatr Clin North Am. 1963;10:499-588.

10. Minich LL, Tani LY, Ritter S, Williams RV, Shaddy RE, Hawkins JA. Usefulness of the preoperative tricuspid/mitral valve ratio for predicting outcome in pulmonary atresia with intact ventricular septum. Am J Cardiol. 2000;85: 1325-8.

11. Alwi M, Choo KK, Latiff HA, Kandavello G, Samion H, Mulyadi MD. Initial results and medium-term follow-up of stent implantation of patent ductus arteriosus in duct-dependent pulmonary circulation. J Am Coll Cardiol. 2004;44: 438-45.

12. Hanley FL, Sade RM, Blackstone EH, Kirklin JW, Freedom RM, Nanda NC Outcomes in neonatal pulmonary atresia with intact ventricular septum: a multi-institutional study. J Thorac Cardiovasc Surg. 1993;105:406-27.

13. Schneider M, Zartner P, Sidiropoulos A, Konertz W, Hausdorf G. Stent implantation of the duct in newborns with duct-dependent circulation. Eur Heart J. 1998;19:1401-9.

14. Behnke IM, Akintuerk H, Thul J, Bauer J, Hagel KJ, Schranz D. Stent implantation in the ductus arteriosus for pulmonary blood supply in congenital heart disease. Catheter Cardiovasc Interv. 2004;61:242-52.

15. Mair DD, Julsrud PR, Puga FJ, Danielson GK. The Fontan procedure for pulmonary atresia with intact ventricular septum: operative and late results. J Am Coll Cardiol. 1997;29:1359-64.

16. Najm HK, Williams WG, Cols JG, Rebeyka IM, Freedom RM. Pulmonary atresia with intact ventricular septum: results of the Fontan procedure. Ann Thorac Surg. 1997;63:669-75

17. Reddy VM, McElhinney DB, Sliverman NH, Marianeschi SM, Hanley FL. Partial biventricular repair for complex congenital heart defects: an intermediate option for complicated anatomy or functionally borderline right complex heart. J Thorac Cardiovasc Surg. 1998;116:21-7.

18. Van Arsdell GS. One and a half ventricle repairs. Semin Thorac Cardiovasc Surg Pediatr Card Surg Annu. 2000;3:173-8.

19. Miyaji K, Shimada M, Sekiguchi A, Ishizawa A, Isoda T, Tsunemoto M. Pulmonary atresia with intact ventricular septum: long-term results of "one and a half ventricular repair." Ann Thorac Surg. 1995;60:1762-4.

20. Shaddy RE, Sturtevant JE, Judd VE, MCGough EC. Right ventricular growth after transventricular pulmonary valvotomy and central aortopulmonary shunt for pulmonary atresia and intact ventricular septum. Circulation. 1990;82(suppl IV): IV157-63.

21. Kouchoukos NT, Blackstone EH, Doty DB, Hanley FL, Karp RB, eds. Cardiac surgery: morphology, diagnostic criteria, natural history, techniques, results, and indication. 3rd ed. Philadelphia: Elsevier; 2003:1095-112.

22. Alwi M, Kandavello G, Choo KK, Bilkis A, Aziz BA, Samion H, et al. Risk factors for augmentation of the flow of blood to the lungs in pulmonary atresia with intact ventricular septum after radiofrequency valvotomy. Cardiol Young. 2005; 15:141-7.

23. Hanseus K, Bjorkhem G, Lundstrom NR, Laurin S. Cross-sectional echocardiographic measurements of right ventricular size and growth in patients with pulmonary atresia and intact ventricular septum. Pediatr Cardiol. 1991;12:135-42.

24. Foker JE, Braunlin EA, St Cyr JA, Hunter D, Molina JE, Moller JH, et al Management of pulmonary atresia with intact ventricular septum. J Thorac Cardiovasc Surg. 1986;92:706-15.

25. Ovaert C, Qureshi SA, Baker EJ, Tynan M. Growth of the right ventricle after successful transcatheter pulmonary valvotomy in neonates and infants with pulmonary atresia and intact ventricular septum. J Thorac Cardiovasc Surg. 1998; 115:1055-62. 